\title{
Trauma Associated Sleep Disorder: A Proposed Parasomnia Encompassing Disruptive Nocturnal Behaviors, Nightmares, and REM without Atonia in Trauma Survivors
}

Study Objectives: To characterize the clinical, polysomnographic and treatment responses of patients with disruptive nocturnal behaviors (DNB) and nightmares following traumatic experiences.

Methods: A case series of four young male, active duty U.S. Army Soldiers who presented with DNB and trauma related nightmares. Patients underwent a clinical evaluation in a sleep medicine clinic, attended overnight polysomnogram (PSG) and received treatment. We report pertinent clinical and PSG findings from our patients and review prior literature on sleep disturbances in trauma survivors.

Results: DNB ranged from vocalizations, somnambulism to combative behaviors that injured bed partners. Nightmares were replays of the patient's traumatic experiences. All patients had REM without atonia during polysomnography; one patient had DNB and a nightmare captured during REM sleep. Prazosin improved DNB and nightmares in all patients. Conclusions: We propose Trauma associated Sleep Disorder (TSD) as a unique sleep disorder encompassing the clinical features, PSG findings, and treatment responses of patients with DNB, nightmares, and REM without atonia after trauma.

Keywords: nightmares, posttraumatic stress disorder, REM sleep behavior disorder, military, veterans

Citation: Mysliwiec V, O'Reilly B, Polchinski J, Kwon HP, Germain A, Roth BJ. Trauma associated sleep disorder: a proposed parasomnia encompassing disruptive nocturnal behaviors, nightmares, and REM without atonia in trauma survivors. J Clin Sleep Med 2014;10(10):1143-1148.
$\mathrm{D}$ isruptive nocturnal behaviors (DNB) consisting of excessive movements, autonomic hyperarousal, abnormal vocalizations and complex motor behaviors, and nightmares which are replays of traumatic experiences are frequently reported sleep disturbances in combat veterans and trauma survivors with and without posttraumatic stress disorder (PTSD). ${ }^{1-6}$ Despite their frequent occurrence, there is no established diagnosis that accurately encompasses these sleep disturbances. Lack of diagnostic criteria is likely secondary to the discrepancy between frequent self-reported DNB and the rare occurrence of DNB in laboratory settings. ${ }^{1,7,8}$ Thus, the exact nature of DNB in terms of their sleep stage, electromyographic (EMG) characteristics, and physiologic parameters are relatively unknown.

Nightmare disorder is reported in up to $80 \%$ of patients with PTSD. ${ }^{9}$ This diagnosis does not acknowledge the presence of the DNB that trauma survivors frequently report. ${ }^{2,4,9}$ Secondary REM behavior disorder is reported to occur in patients with PTSD when REM without atonia (RWA) is present on a polysomnogram (PSG) and dream enactment behaviors are reported or are present on $\mathrm{PSG}^{4,10}$; however, the onset of DNB and nightmares after an inciting traumatic event and the autonomic hyperactivity reported with trauma associated sleep disturbances are clinical and physiologic abnormalities that are not associated with REM behavior disorder (RBD). ${ }^{11-13}$ The inimitable

\section{BRIEF SUMMARY \\ Current Knowledge/Study Rationale: Disruptive nocturnal behaviors and nightmares are frequently reported by trauma survivors and their spouses but rarely documented in laboratory settings. There is no cur- rent diagnosis which encompasses these trauma engendered sleep disturbances. \\ Study Impact: This series of patients describes the clinical, PSG and treatment responses of patients with trauma associated sleep distur- bances. Trauma associated sleep disorder (TSD) is likely a new sleep disorder which can facilitate evaluation and treatment of patients with this constellation of findings.}

characteristics of trauma engendered sleep disturbances have led other authors to suggest that they should be regarded as a distinct nosological entity. $2,14,15$

We report 4 cases of trauma associated sleep disturbances in young active duty U.S. Army soldiers with DNB and nightmares and corresponding PSG findings. This case series illustrates that there is no current diagnosis which accurately encompasses the distinct clinical characteristics, PSG findings, and treatment responses among trauma survivors with DNB and nightmares. We, thus, propose a new diagnostic term, Trauma associated Sleep Disorder (TSD), and review the evidence that supports the hypothesis that this condition may represent a unique trauma related parasomnia. 


\begin{tabular}{|c|c|c|c|c|}
\hline & Case 1 & Case 2 & Case 3 & Case 4 \\
\hline \multicolumn{5}{|l|}{ Demographics } \\
\hline Age & 29 & 34 & 39 & 22 \\
\hline $\mathrm{BMI}$ & 28.2 & 27.2 & 30.1 & 29.5 \\
\hline SSRI usage & Current & Never & Never & Current \\
\hline PTSD diagnosis & Yes & No & No & No \\
\hline \multicolumn{5}{|l|}{ Questionnaires } \\
\hline ESS & 20 & 7 & 12 & 14 \\
\hline ISI & 26 & 20 & 17 & 15 \\
\hline \multicolumn{5}{|l|}{ PSG variables } \\
\hline Sleep efficiency (\%) & 87 & 87 & 93 & 92 \\
\hline WASO (min) & 40.5 & 57 & 19.5 & 34 \\
\hline Sleep latency (min) & 16 & 3 & 12 & 3.5 \\
\hline REM sleep latency (min) & 22 & 97 & 138.5 & 68 \\
\hline Stage 1 sleep (\%) & 6 & 6 & 10 & 10 \\
\hline Stage 2 sleep (\%) & 57 & 33 & 38 & 23 \\
\hline Stage 3 sleep (\%) & 16 & 42 & 31 & 34 \\
\hline REM sleep (\%) & 21 & 20 & 21 & 33 \\
\hline Arousal index & 13 & 14 & 14 & 11 \\
\hline $\mathrm{AHI}$ & 4.1 & 9.8 & 10.6 & 14 \\
\hline PLMS index & 16 & 0 & 0 & 1 \\
\hline PLMS arousal index & 0.3 & 0 & 0 & 0 \\
\hline "Any" EMG activity index a & $17.6 \%$ & $13.7 \%$ & $37.6 \%$ & $14.8 \%$ \\
\hline
\end{tabular}

a "Any" EMG activity index was measured as "any" activity on mentalis muscle EMG per 3-sec mini-epoch and then calculated as percent of total REM. BMI, body mass index; SSRI, selective serotonin reuptake inhibitors; PTSD, posttraumatic stress disorder; ESS, Epworth Sleepiness Scale; ISI, Insomnia Severity Index; WASO, wakefulness after sleep onset; REM, rapid eye movement; AHI, apnea hypopnea index; PLMS, periodic limb movements of sleep.

\section{CASE HISTORIES}

All patients were young men, active duty U.S. Army soldiers, who presented with DNB and nightmares. In each case, the patient reported no sleep disturbances or parasomnias prior to their traumatic experiences. Clinical evaluations occurred in our sleep medicine clinic following referral from either primary care or behavioral health. An attended PSG with video monitoring was performed on each patient in accordance with American Academy of Sleep Medicine standards. ${ }^{16}$ All REM epochs were reviewed by two physicians, board certified in sleep medicine, who assessed the videos for any movements or vocalizations. RWA was quantified utilizing "any" surface EMG activity of the mentalis muscle, according to previously established methods by the SINBAR group and subsequently reported by Sixel-Döring et al. ${ }^{17,18}$ Table 1 summarizes clinical and PSG variables in each case. Case histories and pertinent findings are presented.

\section{Patient 1}

A 29-year-old with combat-related PTSD, insomnia, depression, and alcohol abuse in remission reported sleep disturbances following a 6-month combat-deployment to Afghanistan in 2010. Approximately 12 months after returning home, he developed DNB and nightmares. The DNB consisted of screaming and combative movements. In his nightmares, assailants pursued and threatened him. His medications were paroxetine $30 \mathrm{mg}$ daily for depression; prior to bed he took trazodone 50 $\mathrm{mg}$ for insomnia and prazosin $6 \mathrm{mg}$ for nightmares. He reported some medication non-adherence, but when taking prazosin he had less frequent DNB and nightmares. The DNB and nightmares preceded all medication use. His PSG was conducted 18 months after nightmare and DNB onset. On the night of the PSG, the patient took trazodone $50 \mathrm{mg}$ but failed to take his usual dose of prazosin. Figure 1 depicts a $30-\mathrm{sec}$ epoch of sleep from his early onset REM period. The patient initially had REM with atonia; 34 sec after REM onset, RWA is observed with augmented chin EMG tone and phasic limb movements. Video monitoring documented vocalizations (he repeatedly yelled "oh $\mathrm{f}^{* * *}$, leave me alone!") and defensive limb movements (primarily upper extremity). Tachypnea and tachycardia were associated with his vocalizations. His "any" EMG activity index was $17.6 \%$. Upon completion of the study he recalled a combat-related nightmare, characteristic of his usual nightmares. At clinical follow-up, treatment with image rehearsal therapy (IRT) and cognitive behavioral therapy for insomnia (CBTi) was discussed. He was advised to continue prazosin and implement safe sleeping practices. He was discharged from the Army and did not pursue further medical care.

\section{Patient 2}

A 34-year-old with adjustment disorder, anxiety, insomnia, and symptoms of snoring and witnessed apneas reported sleep disturbances following a 12-month combat-deployment to Iraq in 2003. Upon return from this deployment, he developed difficulties falling and staying asleep; nightmares and night sweats 
Figure 1-PSG epoch from case 1 depicting disruptive nocturnal behaviors in REM sleep and REM without atonia.

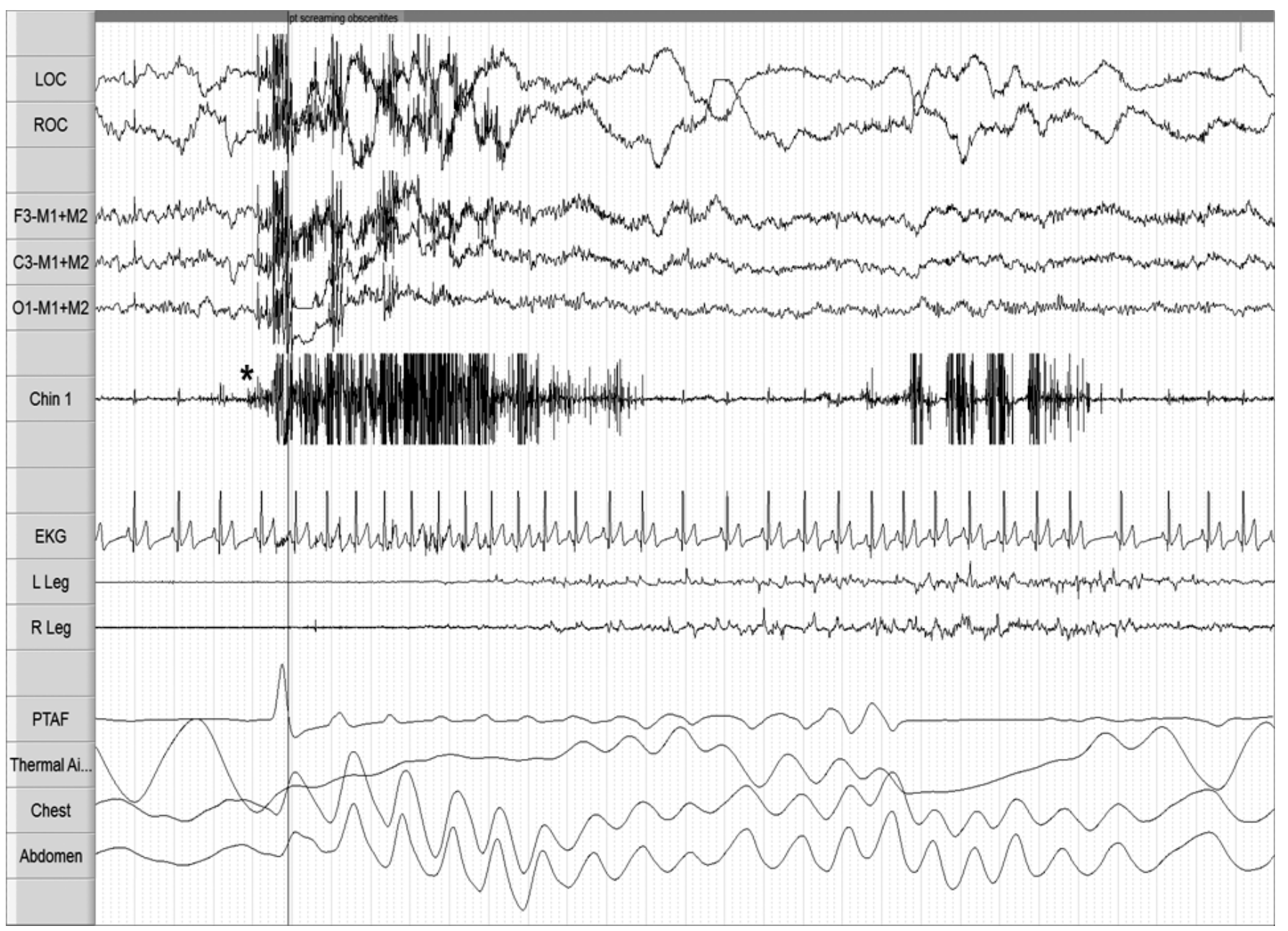

The figure depicts a 30-sec PSG epoch recorded during the first REM period which occurred 22 min after sleep onset. Definitive REMs are observed in the electro-oculography (EOG) channels [see LOC and ROC]. High frequency, low amplitude electroencephalographic (EEG) signal is present in the frontal, central, and occipital EEG channels [see F3-M1+M2, C3-M1+M2, O1-M1+M2]. The initial portion of the epoch demonstrates submental and limb electromyogram (EMG) atonia [see Chin 1 and R\&L Leg], which is consistent with REM sleep, but the later portion of the epoch (following the *) shows elevated submental EMG tone and excessive phasic EMG tone in the limb leads [see Chin 1 and R\&L Leg], consistent with REM without atonia. Tachycardia [see EKG] and tachypnea [see Chest and Abdomen] are present. Technician note annotated onset and video monitoring confirmed vocalizations including profanity and expressions of fear.

started a few months after returning home. He had 2 subsequent deployments and then developed DNB. His DNB consisted of thrashing movements and one episode of choking his wife. Following this violent nocturnal behavior, the patient slept separately from his wife as she feared for her safety. Nightmares involved combat-related experiences. At the time of his sleep evaluation he was taking no medications. His PSG was conducted 11 years after nightmare onset and 6 months after DNB onset. No DNB observed, and the "any" EMG activity index was $13.7 \%$. At clinical follow-up he was treated with CBTi for his insomnia and auto-titrating positive airway pressure (APAP) therapy for mild OSA. For DNB and nightmares he was started on prazosin 30 minutes prior to bedtime and advised to implement safe sleeping practices. Approximately 2 months after starting prazosin at a dose of $4 \mathrm{mg}$, he had complete resolution of his DNB and nightmares, well-treated OSA with adherence to APAP, and overall improved sleep quality. His wife felt safe with him and they resumed sleeping in the same bed.

\section{Patient 3}

A 39-year-old with no significant medical history, but with symptoms of loud, bothersome snoring reported sleep disturbances following a 12-month combat-deployment to Iraq in 2007. His spouse alerted him to DNB which consisted of somnambulism, somniloquy, and combative behaviors of kicking and punching. During these episodes his wife described he looked like he was fighting someone. She reported being struck several times over the years, with one violent nocturnal event that resulted in bruising to her face. This prompted both individuals to seek treatment and the spouse to sleep in a separate room. His nightmares were vague flashbacks to a combat environment with little dream recall upon awakening. His PSG was conducted 6 years after nightmare and DNB onset. No DNB observed, and the "any" EMG activity index was $37.6 \%$. The patient was started on prazosin for DNB and nightmares and APAP for mild OSA. Initially he reported decreased DNB and nightmares with $4 \mathrm{mg}$ prazosin, and his sleep was more 
Table 2-Characteristics of trauma associated sleep disorder compared to REM sleep behavior disorder.

\begin{tabular}{|c|c|c|}
\hline Characteristics & Trauma Associated Sleep Disorder & REM Sleep Behavior Disorder \\
\hline Age & $\begin{array}{l}\text { Typically younger males }{ }^{2} \\
26-55 \text { years of age }\end{array}$ & $\begin{array}{l}\text { Typically older males } \\
36-84 \text { years of age }\end{array}$ \\
\hline Nightmares & Replay of prior traumatic experiences ${ }^{2,3,6,21,22}$ & Defense of the sleeper against attack ${ }^{20}$ \\
\hline Associated illnesses & Post-traumatic stress disorder ${ }^{2,3}$ & $\begin{array}{l}\text { Parkinson's disease } \\
\text { Lewy Body Dementia } \\
\text { Multiple system atrophy } \\
\text { Narcolepsy }^{14,20}\end{array}$ \\
\hline Treatment & $\begin{array}{l}\text { Prazosin }{ }^{9,26} \\
\text { Imagery rehearsal therapy } \\
\text { Clonazepam largely ineffective }{ }^{28}\end{array}$ & Clonazepam ${ }^{12,20}$ \\
\hline
\end{tabular}

restorative. Prazosin was titrated to $12 \mathrm{mg}$ as his DNB persisted in the setting of difficulties with APAP adherence.

\section{Patient 4}

A 22-year-old presented with symptoms of snoring, nocturnal dyspnea, witnessed apneas, and daytime somnolence. He had never deployed but had a traumatic relationship and breakup with a fiancée. Two months after this experience he developed nightmares and DNB. His nightmares occurred almost every night and were related to this relationship. He reported DNB consisting of "screaming, crying, throwing pillows, and cursing" with a witnessed episode where a roommate reported him punching and kicking a wall screaming "I am going to kill you." His new bed partner frequently had bruises and disrupted sleep due to his DNB. After his symptoms started, he was diagnosed with generalized anxiety disorder and treated with sertraline 50 $\mathrm{mg}$. His anxiety improved, but his nightmares and DNB persisted. His PSG was conducted 12 months after symptom onset. No DNB observed, and the "any" EMG activity index was $14.8 \%$. For treatment of mild OSA, APAP was initiated and his sleep disordered breathing and sleepiness resolved with adherence to therapy. His DNB and nightmares persisted, and he was treated with prazosin. At $5 \mathrm{mg}$ his DNB and nightmares completely resolved; however, he had side effects of sleep paralysis and lightheadedness. This resulted in him stopping the prazosin with return of his nightmares and DNB. At $3 \mathrm{mg}$ his nightmares occurred once weekly with resolution of DNB.

\section{DISCUSSION}

This case series reports 4 young male active duty soldiers, with and without PTSD, all with traumatic experiences heralding the onset of DNB and nightmares. All patients had RWA with one patient's PSG capturing a rare in-laboratory nightmare with loud vocalizations, body movements, and sympathetic surges as evidenced by tachypnea and tachycardia. These soldiers are prototypical of the combat veterans seen throughout Department of Defense Medical Treatment Facilities, where there has been a marked increase in the diagnoses of night terrors, nightmare disorder, somnambulism, and RBD. Typically these diagnoses are rendered clinically without PSG documentation. We propose TSD to encompass the complex DNB and nightmares frequently reported, but rarely documented in combat veterans and trauma survivors.

The characteristic PSG finding of TSD is RWA which was present in all of our patients. The patients we report had "any" EMG activity index values ranging from $13.7 \%$ to $37.6 \%$ utilizing the conventional 3 -second mini-epoch scoring. Only patient 3 had an "any" EMG activity index that exceeded the cutoff for both RBD and the $95^{\text {th }}$ percentile of healthy normal reported by the SINBAR group and Frauscher et al. ${ }^{17,19}$ The nature of our report limits our ability to propose an "any" EMG activity index cutoff value for TSD. However, as the majority of our patients had normal values for their "any" EMG activity index, a normal index does not necessarily exclude TSD.

In a study of Vietnam veterans with long-standing PTSD, Ross et al. reported the finding of increased phasic lower extremity EMG tone in REM. ${ }^{15}$ More recently, Wallace et al. reported REM behavior disorder in 4 recent veterans of Operations Iraqi Freedom and Enduring Freedom, all of whom were taking selective serotonin reuptake inhibitors (SSRI) at the time of their PSG. ${ }^{4}$ From this, it appears that RWA may occur more frequently than has been reported in trauma survivors. RWA and dream enactment behaviors have rarely been documented on PSG. ${ }^{3,6}$ In these reports the PSG was typically performed early after the traumatic event. ${ }^{7}$ This is consistent with our 
findings, noting that only patient 1 had DNB and nightmare capture during a PSG.

The findings we report could be interpreted as secondary RBD. However, the clinical characteristics, PSG findings, and responses to treatment support this as a unique sleep disorder (Table 2). Patients with TSD are young males as opposed to patients with RBD who are typically elderly males, many whom have an associated synucleinopathy. ${ }^{12,14,20}$ In RBD, the onset is insidious, occurring without a precipitating event. ${ }^{12,20}$ This contrasts with TSD, where there is a traumatic experience that heralds the onset of DNB and nightmares. In RBD the nightmares develop as part of the disorder, whereas in TSD the nightmares are a replay of the traumatic experience., ,3,6,20-22 $^{\text {The other fea- }}$ ture is the nightmares of RBD tend to have similar themes of self-defense or fending off animals, while the nightmares of TSD are unique to each patient. ${ }^{4,20}$

Many soldiers and their spouses report injuries secondary to DNB, resulting in them sleeping separately to avoid harm. They are surprised when their sleep studies do not document DNB. Lack of DNB capture during PSG despite nightly symptoms at home has been reported in military and non-military PTSD patients. ${ }^{1,7,21,22}$ In patients with RBD, dream enactment behaviors are frequently documented in laboratory settings. ${ }^{12,20}$ A potential reason for lack of PSG documentation of DNB in trauma survivors is the sleep laboratory alters their habitual sleep ritual and the monitored environment is perceived as safe.

The sympathetic activation documented in patient 1 , reported by our patients, along with findings in previous trauma survivors during REM sleep, is unique to TSD. ${ }^{13,15,22}$ In descriptions of RBD by Mahowald and Schenk, there is absence of autonomic reactivity in $\mathrm{RBD}$, specifically the lack of tachycardia despite vigorous limb movements. ${ }^{11,12}$ REM sleep is characterized by marked suppression of sympathetic tone which "may be one of the most fundamental and functionally important aspects of this state." 23 Thus, in TSD there appears to be an overdrive phenomenon, which results in loss of REM atonia and sympathetic suppression.

Two patients we report were taking SSRI, which may have contributed to their RWA. SSRI are associated with early-onset RBD which can persist even after discontinuing the medication. ${ }^{24,25}$ A recent study by Lam et al. concluded that RWA in patients with psychiatric disorders was a complex interaction between their disorder and psychoactive medications, not solely a medication side effect. ${ }^{24}$ Similar to classic RBD, the sleep disturbances in these patients lacked an inciting traumatic event and the traumatic dream replay of TSD. In addition, our patients reported DNB and nightmares prior to initiation of SSRI therapy, making it less likely the etiology of their disorder. The findings by Lam et al. while differing from TSD, support clinical differentiation of patients with RWA co-occurring with psychiatric disorders from RBD.

In the soldier that had his DNB and nightmare captured, this potentially occurred due to lack of prazosin dosing the night of his PSG. Prazosin, an $\alpha_{1}$-adrenergic receptor antagonist has documented efficacy in treating nightmares in PTSD. ${ }^{9,26}$ Prazosin and IRT are the recommended treatments for patients with PTSD-associated nightmares. ${ }^{26,27} \mathrm{We}$ are unaware of any reports of these treatments in specifically ameliorating the DNB that can occur with PTSD; however, in their landmark prazosin
Table 3-Proposed diagnostic criteria for trauma associated sleep disorder.

\section{Proposed Trauma Associated Sleep Disorder Criteria:}

1. Onset after combat or other traumatic experience

2. A history of altered dream mentation that is related to prior traumatic experience

3. Self or witnessed reports of disruptive nocturnal behaviors to include at least one of the following:

a. Abnormal vocalizations

i. Screaming or yelling

b. Abnormal motor behaviors in sleep

i. Tossing, turning or thrashing

ii. Sleep walking

iii. Combative behaviors such as striking bed partner

4. Symptoms of autonomic hyperarousal or PSG monitoring demonstrates one of the following:

a. Tachycardia

b. Tachypnea

c. Diaphoresis

5. REM sleep without atonia present on PSG

a. "Any" EMG activity index may be normal to increased

6. Absence of EEG epileptiform activity on PSG

study, Raskind et al. viewed PTSD nightmares as associated with motor activity. ${ }^{26}$ Clonazepam is the accepted therapy for RBD, ${ }^{11,20}$ and a report of 3 patients with apparent longstanding TSD suggested some efficacy. ${ }^{10}$ A small placebo-controlled study showed clonazepam had no efficacy in patients with findings consistent with TSD. ${ }^{28}$

All the patients we report had clinical improvement with prazosin. Patients 1 and 4 reported return of DNB and nightmares with discontinuation of prazosin. Patients 2 and 4 underwent a follow-up PSG on prazosin and CPAP when their sleep improved. Patient 2 had an "any" EMG activity index of $12.1 \%$ and patient 4 an index of $19.3 \%$. These findings suggest that while prazosin ameliorates clinical symptoms it does not necessarily alter the underlying mechanism of RWA, as neither patient's "any" EMG activity index substantially changed. In addition to prazosin, we recommend patients with TSD receive counseling regarding safe sleeping practices and a sleep medicine consultation to evaluate for other sleep disorders. As all of the patients had insomnia and 3 had OSA, treatment of these comorbid sleep disorders likely contributed to their improved sleep.

In our experience, approximately $50 \%$ of patients with TSD will have PTSD, while the other half will exclusively manifest the nocturnal symptoms of DNB and nightmares. Neuropathologically, RBD has been associated with loss of noradrenergic neurons of the locus ceruleus and/or peri- locus ceruleus, ${ }^{10}$ whereas PTSD appears to be characterized by hyper-adrenergic function of these areas. ${ }^{2,10}$ Blockade of the hyper-adrenergic function is thought to be the primary mechanism of action of prazosin. $^{26}$ There are positron emission tomography (PET) studies that document neural characteristics differentiating RBD and PTSD. These findings may help further explain the clinical differences between RBD and TSD. Cerebral hypometabolism in occipitoparietal, paralimbic, and anterior temporal cortices has been reported on PET scans of patients with RBD 
during wakefulness. ${ }^{29,30}$ In a study of combat-related PTSD with patients who reported DNB, cerebral hypermetabolism was detected in brainstem regions, such as the midbrain reticular formation, locus ceruleus, and the pedunculopontine tegmentum, as well as regions involved in the threat response (e.g., amygdala, dorsal cingulate, and hippocampus) during wakefulness and REM sleep. ${ }^{31}$ Hyperactivity in these neuroanatomical regions during REM may result in RWA and DNB, including dream enactment behaviors.

The present case series and review of sleep disturbances associated with trauma summarizes the features of TSD along with proposed diagnostic criteria (Table 3). The patients we report have multiple factors that potentially contribute to changes in neural modulation of REM atonia and trigger dream enactment. A traumatic experience incites this disorder with contributions from chronic stress, psychiatric and comorbid sleep disorders, and antidepressant use either independently or synergistically facilitating RWA. Our patients' clinical improvement with central noradrenergic blockade suggests prazosin partially normalizes the modulation of REM atonia and may have a role in the treatment of both TSD and RBD. With increasing evidence of an association between trauma and REM sleep pathology, there is a requirement for additional research, which could be wellserved by a clinical registry for trauma survivors, to further establish the characteristics of TSD.

\section{REFERENCES}

1. Germain A, Hall M, Krakow B, Katherine Shear M, Buysse DJ. A brief sleep scale for Posttraumatic Stress Disorder: Pittsburgh Sleep Quality Index Addendum for PTSD. J Anxiety Disord 2005;19:233-44.

2. Mellman TA, Kulick-Bell R, Ashlock LE, Nolan B. Sleep events among veterans with combat-related posttraumatic stress disorder. Am J Psychiatry 1995;152:110-5.

3. van der Kolk B, Blitz R, Burr W, Sherry S, Hartmann E. Nightmares and trauma: a comparison of nightmares after combat with lifelong nightmares in veterans. Am J Psychiatry 1984;141:187-90.

4. Wallace DM, Shafazand S, Ramos AR, et al. Insomnia characteristics and clinical correlates in Operation Enduring Freedom/Operation Iraqi Freedom veterans with post-traumatic stress disorder and mild traumatic brain injury: an exploratory study. Sleep Med 2011;12:850-9.

5. Ross RJ, Ball WA, Sullivan KA, Caroff SN. Sleep disturbance as the hallmark of posttraumatic stress disorder. Am J Psychiatry 1989;146:697-707.

6. Hefez A, Metz L, Lavie P. Long-term effects of extreme situational stress on sleep and dreaming. Am J Psychiatry 1987;144:344-7.

7. Woodward SH, Arsenault NJ, Murray C, Bliwise DL. Laboratory sleep correlates of nightmare complaint in PTSD inpatients. Biol Psychiatry 2000;48:1081-7.

8. American Academy of Sleep Medicine. International Classification of Sleep Disorders. 3rd ed. Darien, IL: American Academy of Sleep Medicine, 2014.

9. Aurora RN, Zak RS, Auerbach SH, et al. Best practice guide for the treatment of nightmare disorder in adults. J Clin Sleep Med 2010;6:389-401.

10. Husain AM, Miller PP, Carwile ST. Rem sleep behavior disorder: potential relationship to post-traumatic stress disorder. J Clin Neurophysiol 2001;18:148-57.

11. Schenck $\mathrm{CH}$, Mahowald MW. Polysomnographic, neurologic, psychiatric, and clinical outcome report on 70 consecutive cases with REM sleep behavior disorder (RBD): sustained clonazepam efficacy in $89.5 \%$ of 57 treated patients. Cleve Clinic J Med 1990;57:S9-S23.

12. Schenck $\mathrm{CH}$, Mahowald MW. REM sleep behavior disorder: clinical, developmental, and neuroscience perspectives 16 years after its formal identification in SLEEP. Sleep 2002;25:120-38.

13. Schlosberg A, Benjamin M. Sleep patterns in three acute combat fatigue cases. J Clin Psychiatry 1978;39:546-9.
14. Manni R, Ratti PL, Terzaghi M. Secondary "incidental" REM sleep behavior disorder: do we ever think of it? Sleep Med 2011;12 Suppl 2:S50-3.

15. Ross RJ, Ball WA, Dinges DF, et al. Motor dysfunction during sleep in posttraumatic stress disorder. Sleep 1994;17:723-32.

16. Berry RB, Brooks R, Gamaldo CE, et al. for the American Academy of Sleep Medicine. The AASM Manual for the Scoring of Sleep and Associated Events: Rules, Terminology and Technical Specifications. Version 2.0. www.aasmnet. org. Darien, IL: American Academy of Sleep Medicine, 2012.

17. Frauscher B, Iranzo A, Gaig C, et al. Normative EMG values during REM sleep for the diagnosis of REM sleep behavior disorder. Sleep 2012;35:835-47.

18. Sixel-Döring F, Trautmann E, Mollenhauer B, Trenkwalder C. Rapid eye movement sleep behavioral events: a new marker for neurodegeneration in early Parkinson disease? Sleep 2014;37:431-8.

19. Frauscher B, Gabelia D, Mitterling T, et al. Motor events during healthy sleep: a quantitative polysomnographic study. Sleep 2014;37:763-73.

20. Olson EJ, Boeve BF, Silber MH. Rapid eye movement sleep behaviour disorder: demographic, clinical and laboratory findings in 93 cases. Brain 2000;123 (Pt 2):331-9.

21. Greenberg R, Pearlman CA, Gampel D. War neuroses and the adaptive function of REM sleep. Br J Med Psychol 1972;45:27-33.

22. Lavie P, Hefez A, Halperin G, Enoch D. Long-term effects of traumatic warrelated events on sleep. Am J Psychiatry 1979;136:175-8.

23. Siegel JM. Mechanisms of sleep control. J Clin Neurophysiol 1990;7:49-65.

24. Lam SP, Li SX, Chan JW, et al. Does rapid eye movement sleep behavior disorder exist in psychiatric populations? A clinical and polysomnographic casecontrol study. Sleep Med 2013;14:788-94.

25. Winkelman JW, James L. Serotonergic antidepressants are associated with REM sleep without atonia. Sleep 2004;27:317-21.

26. Raskind MA, Peskind ER, Hoff DJ, et al. A parallel group placebo controlled study of prazosin for trauma nightmares and sleep disturbance in combat veterans with post-traumatic stress disorder. Biol Psychiatry 2007;61:928-34.

27. Thompson CE, Taylor FB, McFall ME, Barnes RF, Raskind MA. Nonnightmare distressed awakenings in veterans with posttraumatic stress disorder: response to prazosin. J Trauma Stress 2008;21:417-20.

28. Cates ME, Bishop MH, Davis LL, Lowe JS, Woolley TW. Clonazepam for treatment of sleep disturbances associated with combat-related posttraumatic stress disorder. Ann Pharmacother 2004;38:1395-9.

29. Caselli RJ, Chen K, Bandy D, et al. A preliminary fluorodeoxyglucose positron emission tomography study in healthy adults reporting dream-enactment behavior. Sleep 2006;29:927-33.

30. Fujishiro $\mathrm{H}$, Iseki $\mathrm{E}$, Murayama $\mathrm{N}$, et al. Diffuse occipital hypometabolism on [18 F]-FDG PET scans in patients with idiopathic REM sleep behavior disorder: prodromal dementia with Lewy bodies? Psychogeriatrics 2010;10:144-52.

31. Germain A, James J, Insana S, et al. A window into the invisible wound of war: functional neuroimaging of REM sleep in returning combat veterans with PTSD. Psychiatry Res 2013;211:176-9.

\section{ACKNOWLEDGMENTS}

The authors acknowledge Ms. Tristin Baxter, without her administrative excellence this paper would not have occurred. We also acknowledge the service men and women whose personal sacrifices ensure the safety of our country.

\section{SUBMISSION \& CORRESPONDENCE INFORMATION}

Submitted for publication March, 2014

Submitted in final revised form June, 2014

Accepted for publication June, 2014

Address correspondence to: Vincent Mysliwiec, 121st CSH/Brian Allgood Army Community Hospital, Unit \#15244 APO, AP 96205-5244; Tel: 82-010-2706-5511; E-mail: vincent.mysliwiec.mil@mail.mil

\section{DISCLOSURE STATEMENT}

This was not an industry supported study. The authors have indicated no financial conflicts of interest. The opinions and assertions in this manuscript are those of the authors and do not necessarily represent those of the Department of the Army, the Department of Defense, or the U.S. government. 